\title{
How to define successful transition? An exploration of consensus indicators and outcomes in young adults with chronic conditions
}

\author{
J. N. T. Sattoe, S. R. Hilberink and A. van Staa \\ Research Centre Innovations in Care, Rotterdam University of Applied Sciences, Rotterdam, The Netherlands \\ Accepted for publication 17 November 2016
}

Keywords

indicators, transfer, transition, transition outcomes, young adults

Correspondence:

Jane N. T. Sattoe, Research Centre Innovations in Care, Rotterdam University of Applied Sciences, P.O. Box 25035, 3001 HA Rotterdam, The Netherlands

E-mail:

j.n.t.sattoe@hr.nl

\begin{abstract}
Background In this short report, we use data from a previous cohort study to explore the relationship between five out of eight consensus indicators for successful transition and patientreported outcomes in young adulthood.

Methods Data came from a 6-year cohort study that consisted of a survey among 518 young adults with various chronic conditions and a review of their electronic patient records. Associations between five indicators for successful transition and background variables and patient-reported outcomes were explored with Spearman's r. Significant variables were included in stepwise (logistic) regression analyses with transition outcomes as dependent variables.

Results The indicators relate to some extent to better healthcare-related transition outcomes, but not to autonomy in participation. The explained variance of the models varied from $9.7 \%$ to $26.4 \%$. The change in explained variance after adding indicators varied from $2 \%$ to $16 \%$.

Conclusions The challenge of translating the definition of transition into holistic indicators remains. The current consensus indicators are a good start, but there is more to transition than transfer.
\end{abstract}

\section{Introduction}

Over two decades ago, Blum et al. (1993) defined transition in health care as 'a multifaceted, active process that attends to the medical, psychosocial, and educational/vocational needs of adolescents as they move from the child-focused to the adultfocused health-care system', while emphasizing that this 'implies an increase in independent behavior and personal autonomy'. Since then, numerous research articles have been discussing aspects such as transfer readiness of adolescents and the need to improve paediatric practices to support them during their transition and transfer. In the past decade, more attention was paid to collaboration with and practices in adult care and to the study of transition outcomes in young adulthood. More specifically, the question of what constitutes a successful transition has been posed. Recently, Suris and Akre
(2015) conducted a Delphi study to reach international consensus on key elements of transitions programmes and indicators that could serve to assess the success of such programmes.

Given the holistic definition provided by Blum et al. (1993), transition programmes and indicators are expected to relate to the medical, psychosocial and educational/vocational outcomes in young people. In this short report, we use data from a previous cohort study (Sattoe et al. 2014; van Staa \& Sattoe 2014) to explore the relationship between some of the consensus indicators for successful transition and patientreported outcomes in young adulthood. These include outcomes in the medical, psychosocial and educational/ vocational areas, independent behaviour and autonomy in participation. As such, insight could be gained into how current consensus on transition in the international literature 
relates to transition outcomes in the light of the holistic definition of transition (Blum et al. 1993).

\section{Methods}

\section{Study design and participants}

The cohort study included a questionnaire among 518 young adults (YAs; aged 18-25 years) with various chronic conditions and a review of their medical files. Participants of a web-based survey in 2006 (T0) were re-invited for a similar survey 6 years later (T1). In 2006, they were adolescents aged 12 to 18 years and had been undergoing treatment in the Erasmus Medical Center - Sophia's Children's Hospital Rotterdam for more than 3 years. In 2012, death notices and contact information were retrieved from the hospital's electronic patient registry. Eligible YAs were invited through a letter that explained our study and provided a unique password to $\log$ in on a secured website. Reminders were carried out by mail after 1 month and by phone after another month. Respondents were entered in a lottery for 25 cookbooks, 2 smart phones and 1 iPad. The Medical Ethical Committee of the Erasmus Medical Center approved the study (MEC-2012-022).

Of the 1039 participants in the original study, 13 had died and 25 could not be traced. In 2012, 1001 YAs were invited to participate. Of these, 606 responded (60.5\%) while 88 declined to participate. Consequently, 518 YAs submitted the survey (net response rate $51.8 \%$ ). Thirty respondents did not mention their current healthcare provision, 48 were still in paediatric care and five did not provide all necessary information for the current analysis. Hence, the current study sample numbered 433 YAs.

\section{Consensus indicators for successful transition}

Our data allowed for the operationalization of five of the eight indicators (Suris \& Akre, 2015):

- Indicator 1 - patient not lost to follow-up: It is recorded whether a patient is transferred and to where, and/or a note or letter of transfer of the patient to adult care is found in the electronic patient record (EPR) (yes/no). Those who score 'no' are no longer seen in pediatric care, but it is not clear whether and where they receive adult care treatment.

- Indicator 2 - attending scheduled visits in adult care: The patient has not missed any consultations in the 3 years after transfer (yes/no), as reported in the EPR.

- Indicator 3 - patient building a trusting relationship with adult provider: The patient trusts the current adult care provider as indicated by a score $>15$ on a scale of 5-20 (yes/ no) in the survey. A five-item 4-point Likert scale (from $1=$ "never" to $4=$ "always"; $\alpha=0.90$ ) was used. This was measured in the questionnaire with a validated Dutch adaptation of one scale from the American Consumer Assessment of Health Plan Surveys questionnaire (Delnoij et al. 2006).

- Indicator 4 - continuing attention for self-management: The patient reports that professionals give ample attention to self-management topics (including non-medical issues) as indicated by a mean rating on all topics of $\geq 3$ on a Likert scale of $1-5$ (yes/no) in the survey. The topics included relationships and sexuality and subjects related to the prospects like family planning, further education, jobs and the chronic condition (Van Staa et al. 2015).

- Indicator 5 - patient and family satisfaction with transfer of care: The patient is satisfied with the process of transfer as indicated by a score of $\geq 7$ on a 1-10 numeric scale (yes/no). No information on family satisfaction was available.

\section{Background variables}

Patient characteristics were age, gender, age at transfer, educational level (low/medium vs. high) and presence of a physical limitation (yes/no) (Sattoe et al. 2014; van Staa \& Sattoe 2014).

\section{Patient-reported transition outcomes}

Transition outcomes were

- Adherence to therapeutic regimen, measured with the fiveitem Medication Adherence Report Scale [5-point Likert scales (from $1=$ "never" to $5=$ "always"; $\alpha=0.75$ )] (Horne \& Hankins, unpubl. observ.).

- Disease-related self-efficacy, measured with the 16-item On Your Own Feet Self-efficacy Scale [4-point Likert scales (from $1=$ "no, definitely not" to $4=$ "yes, certainly"; $\alpha=0.87$ )] (van Staa 2012).

- Independence during consultations, self-rated on a 1-10 numeric scale.

- Actual independent behaviours during consultations, measured with the seven-item Independent Behaviors During Consultations Scale [5-point Likert scales (from 1= "never" to $5=$ "always"; $\alpha=0.79$ )] (van Staa \& Sattoe 2014).

- Health-related quality of life (HRQoL), measured with the 37item DISABKIDS questionnaire [5-point Likert scales (from $1=$ "often" to $5=$ "never"; $\alpha=0.95$ )] (Simeoni et al. 2007). 
- Perceived impact of the chronic condition on vocational functioning, measured with a five-item scale (5-point Likert scales [from $1=$ "totally disagree" to $5=$ "totally agree"; $\alpha=0.89$ )] (Sattoe et al. 2014).

- Autonomy in social participation in seven life areas; measured with the Rotterdam Transition Profile (Donkervoort et al. 2009). The Rotterdam Transition Profile describes participation in seven life areas defined in the International Classification of Functioning, Disability, and Health: finances, employment and education, housing, intimate relationships, sexuality, transportation and leisure. We dichotomized the outcomes, a score of 1 indicated full autonomy in participation.

\section{Analysis}

Associations between indicators for successful transition and background variables and patient-reported outcomes were explored with Spearman's r. Significant variables were included in stepwise (logistic) regression analyses with the transition outcomes as dependent variables. The first step included background variables; the second step added the indicators. Backward logistic analysis was used to test if background and outcome variables were associated with being included in the regression analysis or not. Data were analysed with SPSS 20 .

\section{Results}

Characteristics of the current study sample and scores per indicator are presented in Table 1. In the study sample, 73.7\% of the YAs were not lost to follow-up after transfer (indicator 1; i.e. a transfer note was available in the chart) and $87.4 \%$ attended all scheduled visits in adult care in the 3 years after transfer (indicator 2). Less than half of the YAs (45.2\%) reported continuing attention for self-management after transfer (indicator 3), 66.2\% built a trusting relationship with their adult care provider (indicator 4), and $64.5 \%$ were satisfied with the transfer of care (indicator 5) (Table 1). In bivariate correlation analysis, the indicators were not associated with educational level, self-reported adherence and autonomy in social participation on all life areas except the financial domain. Associations with the latter were nonsignificant in the regression analysis. Because of the large number of missing data in indicators 2 and 4 , the multivariate models included 178 cases. Backward logistic regression showed that the YAs without any missing data were older
Table 1. Characteristics of the study sample and scores per indicator $(n=433)$

\begin{tabular}{|c|c|}
\hline \multicolumn{2}{|l|}{ Characteristics } \\
\hline Age $[$ mean $\pm S D]$ & $20.76 \pm 1.88$ \\
\hline Gender (male) $[n(\%)]$ & $161(37.2)$ \\
\hline Level of education (high) $[n(\%)]^{a}$ & $192(45.4)$ \\
\hline Age at transfer $[\text { mean } \pm \mathrm{SD}]^{\mathrm{b}}$ & $17.45 \pm 2.00$ \\
\hline Physical limitation (yes) $[n(\%)]^{c}$ & $124(28.8)$ \\
\hline \multicolumn{2}{|l|}{ Consensus indicators } \\
\hline $\begin{array}{l}\text { Indicator } 1-\text { patient not lost to } \\
\text { follow-up (yes) }[n(\%)]\end{array}$ & $319(73.7)$ \\
\hline $\begin{array}{l}\text { Indicator } 2 \text { - attending scheduled } \\
\text { visits in adult care (yes) }[n(\%)]^{d}\end{array}$ & $216(87.4)$ \\
\hline $\begin{array}{l}\text { Indicator } 3 \text { - patient building a trusting } \\
\text { relationship with adult provider (yes) }[n(\%)]^{\mathrm{e}}\end{array}$ & $215(66.2)$ \\
\hline $\begin{array}{l}\text { Indicator } 4 \text { - continuing attention for } \\
\text { self-management after transfer (yes) }[n(\%)]^{\mathrm{e}}\end{array}$ & $147(45.2)$ \\
\hline $\begin{array}{l}\text { Indicator } 5 \text { - patient satisfaction with } \\
\text { transfer of care (yes) }[n(\%)]^{f}\end{array}$ & $267(64.5)$ \\
\hline \multicolumn{2}{|l|}{${ }^{a} n=10$ missing; } \\
\hline \multicolumn{2}{|l|}{${ }^{b} n=30$ missing; } \\
\hline \multicolumn{2}{|l|}{ ' $n=3$ missing; } \\
\hline \multicolumn{2}{|c|}{$\begin{array}{l}n=186 \text { missing (because only young people who transferred } 3 \text { years or } \\
\text { longer ago at time of the chart review were included, and these data were }\end{array}$} \\
\hline
\end{tabular}

and were transferred at younger age compared with YAs with missing data, but no associations with any transition outcomes appeared.

Not being lost to follow-up (indicator 1) was negatively associated with disease-related self-efficacy, while building a trusting relationship with the adult provider and patient satisfaction with the transfer of care (indicators 3 and 5) had positive associations with self-efficacy. Not attending all scheduled visits in adult care (indicator 2) was negatively associated with disease-related self-efficacy and self-rated independence and independent behaviours during consultations. Continuing attention for self-management in adult care (indicator 4) was positively associated with HRQoL and negatively with perceived impact of the chronic condition on vocational functioning (i.e. with less impact). The explained variance of the models varied from $9.7 \%$ to $26.4 \%$. The change in explained variance after adding indicators in step 2 varied from $2 \%$ to $16 \%$ (Table 2 ).

\section{Discussion}

Continuing attention for self-management in adult care (indicator 4) was the only indicator associated with higher HRQoL and less perceived impact on vocational functioning. HRQoL is considered an important patient-reported outcome 
Table 2. Results of regression analyses $(n=178)$

Disease-related self-efficacy

Step 1: explained variance $R^{2}=0.10 ; F$ value (d.f.): $4.78(4,173) ; P=0.001$ Age

Gender (male)

Physical limitation (yes)

Step 2: Explained variance: $R^{2}=0.26 ; F$ value (d.f.): $6.70(9,168) ; P<0.001$ Indicator 1 - patient not lost to follow-up

Indicator 2 - attending scheduled visits in adult care

Indicator 3 - patient building a trusting relationship with adult provider

Indicator 4 - continuing attention for self-management

Indicator 5 - patient and family satisfaction with transfer of care

Independence during consultations

Step 1: explained variance $R^{2}=0.07 ; F$ value (d.f.): $3.37(4,173) ; P=0.011$

Age

Gender (male)

Age at transfer

Physical limitation (yes)

Step 2: explained variance: $R^{2}=0.15 ; F$ value (d.f.): $3.21(9,168) ; P=0.001$

Indicator 1 - patient not lost to follow-up

Indicator 2 - attending scheduled visits in adult care

Indicator 3 - patient building a trusting relationship with adult provider

Indicator 4 - continuing attention for self-management

Indicator 5 - patient satisfaction with transfer of care

Independent behaviours during consultations

Step 1: explained variance $R^{2}=0.029 ; F$ value (d.f.): $1.28(4,173) ; P=0.280$

Age

Gender (male)

Age at transfer

Physical limitation (yes)

Step 2: explained variance: $R^{2}=0.097 ; F$ value (d.f.): $2.00(9,168) ; P=0.041$ Indicator 1 - patient not lost to follow-up

Indicator 2 - attending scheduled visits in adult care

Indicator 3 - patient building a trusting relationship with adult provider Indicator 4 - continuing attention for self-management

Indicator 5 - patient satisfaction with transfer of care

Health-related quality of life

Step 1: explained variance $R^{2}=0.12 ; F$ value (d.f.): $5.69(4,173) ; P<0.001$

Age

Gender (male)

Age at transfer

Physical limitation (yes)

Step 2: explained variance: $R^{2}=0.21 ; F$ value (d.f.): $4.94(9,168) ; P<0.001$ Indicator 1 - patient not lost to follow-up

Indicator 2 - attending scheduled visits in adult care

Indicator 3 - patient building a trusting relationship with adult provider Indicator 4 - continuing attention for self-management

Indicator 5 - patient satisfaction with transfer of care

Perceived impact of the chronic condition on vocational functioning

Step 1: explained variance $R^{2}=0.07 ; F$ value (d.f.): $3.50(4,175) ; P=0.009$

Age

Gender (male)

Age at transfer

Physical limitation (yes)

Step 2: explained variance: $R^{2}=0.14 ; F$ value (d.f.): $2.97(9,170) ; P=0.003$

Indicator 1 - patient not lost to follow-up
0.34

0.03

$-0.27$

$-0.14$

0.001

0.62

0.009

0.04

0.04

0.04

0.001

0.06

0.005

0.29

$-0.11$

0.01

0.15

$-0.24$

$-0.13$

0.03

0.08

$-0.04$

$-0.17$

0.14

0.07

0.11

0.60

0.02

0.06

0.37

0.15

0.11

0.33

$-0.15$

$-0.07$

$-0.04$

0.06

0.54

0.59

$-0.03$

0.74

$-0.22$

0.06

0.00

0.005

0.46

0.98

0.14

0.08

0.13

0.15

$-0.23$

$-0.22$

0.22

0.05

0.03

0.002

0.03

0.66

0.11

0.20

0.003

0.22

0.13

0.07

0.02

0.82

$-0.02$

0.76

0.14

0.76
0.22

0.19

0.01

$-0.00$

0.99 
Table 2. (Continued)

\begin{tabular}{|c|c|c|}
\hline Model & Standardized betas $(\beta)$ of step 2 & $P$ value \\
\hline Indicator 2 - attending scheduled visits in adult care & -0.11 & 0.16 \\
\hline Indicator 3 - patient building a trusting relationship with adult provider & -0.03 & 0.66 \\
\hline Indicator 4 - continuing attention for self-management & -0.22 & 0.004 \\
\hline Indicator 5 - patient and family satisfaction with transfer of care & -0.06 & 0.42 \\
\hline $\begin{array}{l}\text { Autonomy in social participation, financial domain } \\
\text { Step 1: explained variance } R^{2}=0.21 ; \chi^{2}=31.33 \text {, d.f. }=4 ; P<0.001\end{array}$ & Odds ratio $(\mathrm{Cl})$ of step 2 & $P$ value \\
\hline Age & $1.67(1.22-2.29)$ & 0.001 \\
\hline Gender (male) & $0.77(0.37-1.60)$ & 0.48 \\
\hline Age at transfer & $0.95(0.69-1.31)$ & 0.75 \\
\hline Physical limitation (yes) & $2.78(1.36-5.71)$ & 0.005 \\
\hline \multicolumn{3}{|l|}{ Step 2: explained variance: $R^{2}=0.23 ; \chi^{2}=33.93$, d.f. $=9 ; P<0.001$} \\
\hline Indicator 1 - patient not lost to follow-up & $1.26(0.52-3.03)$ & 0.60 \\
\hline Indicator 2 - attending scheduled visits in adult care & $0.80(0.31-2.07)$ & 0.65 \\
\hline Indicator 3 - patient building a trusting relationship with adult provider & $0.73(0.35-1.51)$ & 0.39 \\
\hline Indicator 4 - continuing attention for self-management & $1.32(0.67-2.57)$ & 0.42 \\
\hline Indicator 5 - patient satisfaction with transfer of care & $1.48(0.74-2.98)$ & 0.27 \\
\hline
\end{tabular}

as well as a relevant transition outcome (Fair et al. 2016). Still, receiving continuing attention for self-management was reported by less than half of the sample. This finding highlights the challenge for adult care to incorporate a more holistic approach to care delivery for YAs with chronic conditions. Moreover, the international transition community seems to be facing this challenge, too, because all other consensus indicators concern continuity of care and healthcare-related outcomes (Suris \& Akre, 2015). It therefore comes as no surprise that the indicators explained some of the variance of healthcare-related outcomes and none of the variance in social participation. Thus, when using these indicators to define successful transition, most emphasis will be placed on the medical aspect, i.e. the transfer of care, and much less on young people's successful transition to adulthood (i.e. reaching their full potential). This narrow focus does not seem to be justified, because the transfer of care is influenced by the transition to adulthood (Sattoe 2015). Moreover, young people themselves repeatedly stress the importance of non-medical themes when asked about their lived experiences (Taylor et al. 2008). The results suggest that the consensus indicators cover the medical aspects of transition, but do not address the psychosocial and participation outcomes. So, although the holistic definition of transition seems to be leading for over 20 years, the challenge of translating it into holistic indicators and practice remains. The current consensus indicators are a good start, but there is more to transition than transfer.

Two limitations of this study must be considered. First, loss to follow-up (indicator 1) can be operationalized in different ways. We used data from the EPR, whether the transfer to adult care was recorded and where young people were transferred to. It cannot be ruled out that YAs, classified as 'lost to follow-up', are seen in adult care without knowledge of their paediatric care provider. Still, a comparison of the EPR data about referrals with patient-reported data (from the questionnaire) on current providers confirmed that a quarter of young people were not in regular (specialist) care anymore $(n=125 ; 25.6 \%)$. Agreement on the current provider between EPR and patient-reported data was only found in 293 out of 488 cases (60.0\%). Kappa was 0.40 (95\% CI, 0.34-0.46), indicating 'fair' agreement. Most disagreement was found in the group of YAs stating they are 'not in active treatment anymore'.

Finally, indicator 4 had many missing values on the scale measuring continuing attention for self-management. As this is the only indicator related to HRQoL and vocational functioning, we compared the cases with missing values with those without missing values on these outcome measures. Our additional analysis showed that the group without missing values reported lower HRQoL and more perceived impact on vocational functioning. This might have influenced the score of the study sample on indicator 4 , but we think the associations found still emphasize the need for a broader view on transition.

\section{Key messages}

- The indicators relate to some extent to better healthcarerelated transition outcomes, but not to more autonomy in participation. Additional indicators are needed to encourage healthcare services to cover both the medical and non-medical challenges that adolescents and YAs face and to assess the success of more holistic transition programmes. 


\section{Funding}

The On Your Own Feet programme (2004-2008) was funded by ZonMw, The Netherlands Organization for Health Research and Development (grant 32560005) and the Dutch charity foundation Kinderpostzegels (grant 23674/1/4). The follow-up study was performed in the framework of the Selfmanagement and Participation Innovation Lab, supported by SIA-RAAK, the Foundation Innovation Alliance with funding from the Dutch Ministry of Education, Culture and Science (OCW) (PRO-02-025).

\section{Conflict of interests}

The funding sources had no role in the design and conduct of the study; collection, management, analysis and interpretation of the data; preparation, review or approval of the manuscript; and decision to submit the manuscript for publication.

\section{Acknowledgements}

The authors thank the other members of the On Your Own Feet Research Group, Jos Latour, Susan Jedeloo and Linda van der Knaap, for their support in the design and execution of the study. Laila Oumansour, Wendy de Boer, Marjolijn Bal and Mariëlle Peeters are thanked for their assistance in collecting data.

\section{References}

Blum, R. W., Garell, D., Hodgman, C. H., Jorissen, T. W., Okinow, N. A., Orr, D. P. \& Slap, G. B. (1993) Transition from child-centered to adult health-care systems for adolescents with chronic conditions: a position paper of the Society for Adolescent Medicine. Journal of Adolescent Health, 14, 570-576.

Delnoij, D. M., Ten Asbroek, G., Arah, O. A., De Koning, J. S., Stam, P., Poll, A., ... \& Klazinga, N. S. (2006). Made in the USA: the import of American Consumer Assessment of Health Plan Surveys $\left(\right.$ CAHPS $\left.^{\circledast}\right)$ into the Dutch social insurance system. The European Journal of Public Health, 16, 652-659.

Donkervoort, M., Wiegerink, D. J., Van Meeteren, J., Stam, H. J. \& Roebroeck, M. E. (2009) Transition to adulthood: validation of the Rotterdam Transition Profile for young adults with cerebral palsy and normal intelligence. Developmental Medicine \& Child Neurology, 51, 53-62.

Fair, C., Cuttance, J., Sharma, N., Maslow, G., Wiener, L., Betz, C., Porter, J., McLaughlin, S., Gilleland-Marchak, J., Renwick, A., Naranjo, D., Jan, S., Javalkar, K. \& Ferris, M. (2016) International and interdisciplinary identification of health care transition outcomes. JAMA Pediatrics, 170, 205-211.

Sattoe, J.N.T. (2015). Growing up with a chronic condition: challenges for self-management and self-management support. $\mathrm{PhD}$ thesis. Rotterdam: Erasmus University Rotterdam.

Sattoe, J. N. T., Hilberink, S. R., van Staa, A. \& Bal, R. (2014) Lagging behind or not? Four distinctive social participation patterns among young adults with chronic conditions. Journal of Adolescent Health, 54, 397-403.

Simeoni, M. C., Schmidt, S., Muehlan, H., Debensason, D., Bullinger, M. \& DISABKIDS Group (2007) Field testing of a European quality of life instrument for children and adolescents with chronic conditions: the 37-item DISABKIDS chronic generic module. Quality of Life Research, 16, 881-893.

Suris, J. C. \& Akre, C. (2015) Key elements for, and indicators of, a successful transition: an international Delphi study. Journal of Adolescent Health, 56, 612-618.

Taylor, R. M., Gibson, F. \& Franck, L. S. (2008) The experience of living with a chronic illness during adolescence: a critical review of the literature. Journal of Clinical Nursing, 17, 3083-3091.

van Staa, A. L. \& Sattoe, J. N. T. (2014) Young adults' experiences and satisfaction with the transfer of care. Journal of Adolescent Health, 55, 796-803.

van Staa, A.L. (2012). On your own feet: adolescents with chronic conditions and their preferences and competencies for care. $\mathrm{PhD}$ Thesis. Rotterdam: Erasmus University Rotterdam.

van Staa, A. L., Sattoe, J. N. T. \& Strating, M. M. (2015) Experiences with and outcomes of two interventions to maximize engagement of chronically Ill adolescents during hospital consultations: a mixed methods study. Journal of Pediatric Nursing, 30, 757-775. 